\title{
DESENVOLVIMENTO DE METODOLOGIA PARA PREVISÃO DA EFICIÊNCIA DE DESFOSFORAÇÃO DE AÇO UTILIZANDO A TERMODINÂMICA COMPUTACIONAL*
}

\author{
Fernando Dellacqua Cristo ${ }^{1}$ \\ Silas Gambarine Soares ${ }^{1}$ \\ Sábata Marla Reis Durães Oliveira \\ Heitor Cristo Clem de Oliveira ${ }^{1}$ \\ José Roberto de Oliveira ${ }^{2}$
}

\section{Resumo}

Durante o processo de desfosforação, as principais fases sólidas formadas na escória são o silicato dicálcico $\left(2 \mathrm{CaO} . \mathrm{SiO}_{2}\right)$ e tricálcio $\left(3 \mathrm{CaO} . \mathrm{SiO}_{2}\right)$, o fosfato tricálcico $\left(3 \mathrm{CaO} . \mathrm{P}_{2} \mathrm{O}_{5}\right)$, e o $3 \mathrm{CaOSiO}_{2} \cdot 3 \mathrm{CaOP}_{2} \mathrm{O}_{5}$ formado pela oxidação do fósforo em solução sólida com o silicato tricálcio. O objetivo deste trabalho é estudar a formação destes compostos e avaliar a influência das fases sólidas e líquidas formadas na eficiência da desfosforação. Foram analisadas diferentes composições de escórias industriais com diferentes teores de $\mathrm{CaO}$ e FeO. As fases presentes na escória e a sua viscosidade foram determinadas pela termodinâmica computacional utilizando o software FactSage (usando os bancos de dados GTOXID e FTOXID) e os resultados destas análises foram relacionados com a eficiência de desfosforação. A eficiência da desfosforação também foi relacionada com os diferentes modelos de relação de partição do fósforo (Lp) disponíveis na literatura.

Palavras-chave: Desfosforação; Termodinâmica computacional; Factsage.

\section{DEVELOPMENT OF METHODOLOGY FOR FORECAST OF STEEL DEPHOSPHORIZATION EFFICIENCY USING COMPUTATIONAL THERMODYNAMICS}

\section{Abstract}

During the process of dephosphorization, the main solid phases formed in slag is dicalcium silicate $\left(2 \mathrm{CaO} . \mathrm{SiO}_{2}\right)$ and tricalcium silicate $\left(3 \mathrm{CaO} . \mathrm{SiO}_{2}\right)$, the tricalcium phosphate $\left(3 \mathrm{CaO} \cdot \mathrm{P}_{2} \mathrm{O}_{5}\right)$, and the $3 \mathrm{CaOSiO}_{2} \cdot 3 \mathrm{CaOP}_{2} \mathrm{O}_{5}$. formed by the oxidation of phosphorus in solid solution with the tricalcium silicate. The aim of this work is study the formation of these compounds and evaluate the influence of solid and liquid phases formed in the efficiency of steel dephosphorization. Different compositions of industrial slags with different $\mathrm{CaO}$ and $\mathrm{FeO}$ contents were analyzed. The present phases in the slag and its viscosity were determined by computational thermodynamics using the software FACTSAGE (using GTOXID and FTOXID database) and the results of these analyzes was related to the dephosphorization efficiency obtained by different slag. The dephosphorization efficiency was also related to the different models of equilibrium phosphorus partition ratio (Lp) available in the literature.

Keywords: Dephosphorization; Computational thermodynamics; Factsage.

1 Eng. Metalurgista, Mestrando em Engenharia Metalúrgica e de Materiais, Instituto Federal do Espírito Santo (IFES), Vitória, ES, Brasil.

2 Prof. Dr., cursos de graduação e pós-graduação em Engenharia Metalúrgica e de Materiais, Instituto Federal do Espírito Santo (IFES), Vitória, ES, Brasil. 


\section{INTRODUÇÃO}

Os mecanismos de transferência do fósforo do metal para a escória em aciarias a oxigênio ainda é bastante discutido devido à complexidade de fenômenos que ocorrem durante a conversão do gusa em aço. Isto está relacionado, principalmente, com a variação da composição química da escória durante o período de sopro. De forma a compreender melhor a remoção do fósforo, parâmetros como o Coeficiente de Partição do Fósforo (Lp) e Coeficiente de atividade do $\mathrm{P}_{2} \mathrm{O}_{5}$ ( $\gamma_{\mathrm{P} 2 \mathrm{O}}$ ), dentre outros, são frequentemente utilizados para avaliar a eficiência de desfosforação, cujas as Equações 1 e 2 a seguir:

$$
\begin{gathered}
L p=\frac{\%(P)_{\text {Escória }}}{\%[P]_{\text {Metal }}} \\
\gamma_{P_{2} O_{5}}=\frac{a_{P_{2} O_{5}}}{N_{P_{2} O_{5}}}
\end{gathered}
$$

A seguir, são listados alguns modelos desenvolvidos com base na termodinâmica clássica para determinação do coeficiente de partição do fósforo e coeficiente de atividade do $\mathrm{P}_{2} \mathrm{O}_{5}$ :

- Turkdogan [1]:

$$
\log (L p)=\frac{21740}{T}+0,071\left[(\% C a O+0,3 \% M g O)+\left(\% C a F_{2}\right)\right]-9,87+2,5 \log [\% O]
$$

Onde:

$$
\begin{aligned}
& {[\% \mathrm{O}]=(0,1 \mathrm{~T}-155,3) \cdot 10^{-4} \cdot(\% \mathrm{FeO})} \\
& \mathrm{BO}=(\% \mathrm{CaO})+\left(\% \mathrm{CaF}_{2}\right)+0,3(\% \mathrm{MgO})
\end{aligned}
$$

- Turkdogan [2]:

$$
\log \gamma_{P_{2} \mathrm{O}_{5}}=-9,84-0,142\{(\% \mathrm{CaO})+0,3(\% \mathrm{MgO})\}
$$

Neste contexto, o estudo de características das escórias, como a viscosidade e as fases presentes se torna importante, pois, com a mudança destas características, o mecanismo de desfosforação pode ser alterado, influenciando diretamente na eficiência do processo de desfosforação. A exemplo disso, uma série de trabalhos têm sido realizados com o intuito de verificar a influência que a presença de fases sólidas na escória tem no mecanismo de remoção do fósforo [3,4]. Sabe-se que a presença de cal e magnésia livre, $\mathrm{CaO}_{(\mathrm{s})}$ e $\mathrm{MgO}_{(\mathrm{s})}$, assim como soluções sólidas de $\mathrm{CaO}$ e $\mathrm{MgO}$, aumentam de forma considerável a viscosidade da escória, além de causar variação significativa no índice de espumação da escória durante o período de sopro [5]. Estes fatores, fisicamente, provocam instabilidade no volume da escória, tendo como consequência o descontrole da reação de desfosforação. $O$ silicato dicálcio $\left(2 \mathrm{CaO} . \mathrm{SiO}_{2}\right)$, segundo Miyamoto [6], é capaz de formar solução sólida de $3 \mathrm{CaO} . \mathrm{P}_{2} \mathrm{O}_{5}$ e $2 \mathrm{CaO} . \mathrm{SiO}_{2}$, mantendo grande parte do fósforo condensado em fase sólida, diminuindo assim o teor de fósforo na escória líquida, possibilitando aumentar a capacidade de desfosforação da escória. 
Outra fase sólida comentada na literatura é o silicato tricálcio $\left(3 \mathrm{CaO} . \mathrm{SiO}_{2}\right)$, por ter efeito negativo na dissolução da cal. Este por sua vez, pode formar uma espécie de cluster ao redor das partículas de cal, com alto ponto de fusão. Isto provoca a diminuição do rendimento da cal e prejuízos à desfosforação.

Bardenheuer [7] realizaram experimentos para avaliar o coeficiente de partição do fósforo em função da composição de escórias do sistema $\mathrm{CaO}-\mathrm{FeO}-\mathrm{SiO}_{2}$ e observaram que o menor valor de partição correspondia à interface entre a fase líquida da escória e o silicato tricálcio.

\section{MATERIAIS E MÉTODOS}

Com base nos dados de 16 corridas realizadas nos convertedores da Arcelor Mittal Tubarão, foram realizadas simulações com o software de termodinâmica computacional Factsage utilizando dois bancos de dados deste programa o FTOXID e o GTOXID, afim de obter informações que possam ser úteis no estudo da desfosforação. Os dados das corridas são descritos nas Tabelas 1, 2 e 3.

Tabela 1. Composição química do gusa carregado no convertedor

\begin{tabular}{ccccccc}
\hline Corrida & $\mathbf{C}$ & $\mathbf{S i}$ & $\mathbf{M n}$ & $\mathbf{P}$ & $\mathbf{S}$ & Tgusa $\left({ }^{\circ} \mathbf{C}\right)$ \\
\hline 1 & 4,86 & 0,39 & 0,42 & 0,074 & 0,001 & 1418 \\
2 & 4,79 & 0,43 & 0,42 & 0,078 & 0,001 & 1393 \\
3 & 4,8 & 0,45 & 0,42 & 0,078 & 0,001 & 1398 \\
4 & 4,55 & 0,26 & 0,34 & 0,083 & 0,001 & 1348 \\
5 & 4,61 & 0,2 & 0,33 & 0,076 & 0,001 & 1314 \\
6 & 4,82 & 0,38 & 0,4 & 0,077 & 0,005 & 1425 \\
7 & 4,68 & 0,62 & 0,47 & 0,089 & 0,001 & 1363 \\
8 & 4,45 & 0,78 & 0,49 & 0,097 & 0,002 & 1255 \\
9 & 4,55 & 0,25 & 0,36 & 0,085 & 0,001 & 1301 \\
10 & 4,81 & 0,26 & 0,43 & 0,08 & 0,002 & 1390 \\
11 & 4,52 & 0,59 & 0,44 & 0,092 & 0,002 & 1255 \\
12 & 4,88 & 0,31 & 0,4 & 0,074 & 0,001 & 1352 \\
13 & 4,68 & 0,38 & 0,39 & 0,078 & 0,001 & 1328 \\
14 & 4,92 & 0,26 & 0,41 & 0,073 & 0,002 & 1402 \\
15 & 4,66 & 0,21 & 0,32 & 0,073 & 0,001 & 1356 \\
16 & 4,71 & 0,17 & 0,36 & 0,075 & 0,001 & 1312 \\
\hline Média & 4,71 & 0,37 & 0,40 & 0,080 & 0,0015 & 1350,6 \\
\hline
\end{tabular}


Tabela 2. Composição química do aço no fim de sopro em convertedor

\begin{tabular}{cccccccc}
\hline Número & $\mathbf{C}$ & $\mathbf{S i}$ & $\mathbf{M n}$ & $\mathbf{P}$ & $\mathbf{S}$ & $\begin{array}{c}\text { Tfs } \\
\left({ }^{\circ} \mathbf{C}\right)\end{array}$ & $\begin{array}{c}\text { Peso } \\
\text { Aço(ton) }\end{array}$ \\
\hline 1 & 0,044 & 0,001 & 0,108 & 0,011 & 0,0057 & 1677 & 310,8 \\
2 & 0,038 & 0,002 & 0,114 & 0,013 & 0,006 & 1675 & 312,7 \\
3 & 0,038 & 0,001 & 0,097 & 0,011 & 0,0064 & 1673 & 309,5 \\
4 & 0,051 & 0,001 & 0,119 & 0,015 & 0,0041 & 1667 & 314,9 \\
5 & 0,04 & 0,001 & 0,098 & 0,015 & 0,005 & 1665 & 312,8 \\
6 & 0,047 & 0,002 & 0,116 & 0,012 & 0,0037 & 1664 & 317,4 \\
7 & 0,034 & 0,001 & 0,063 & 0,014 & 0,004 & 1684 & 297,7 \\
8 & 0,038 & 0,001 & 0,064 & 0,012 & 0,0043 & 1677 & 285,3 \\
9 & 0,032 & 0,001 & 0,082 & 0,012 & 0,0054 & 1670 & 318,2 \\
10 & 0,107 & 0,001 & 0,129 & 0,013 & 0,0044 & 1669 & 310,2 \\
11 & 0,094 & 0,002 & 0,135 & 0,013 & 0,0038 & 1670 & 298,9 \\
12 & 0,037 & 0,001 & 0,127 & 0,018 & 0,0044 & 1685 & 315,5 \\
13 & 0,037 & 0,001 & 0,106 & 0,012 & 0,0043 & 1681 & 305,6 \\
14 & 0,039 & 0,001 & 0,116 & 0,014 & 0,0051 & 1660 & 313,2 \\
15 & 0,044 & 0,002 & 0,102 & 0,016 & 0,0054 & 1675 & 312,6 \\
16 & 0,028 & 0,001 & 0,073 & 0,016 & 0,0051 & 1673 & 300,6 \\
\hline Média & 0,047 & 0,001 & 0,103 & 0,014 & 0,0048 & 1673 & 308,5 \\
\hline
\end{tabular}

Tabela 3. Composição química da escória no fim de sopro em convertedor

\section{Composição Escória Fim de Sopro}

\begin{tabular}{cccccccccc}
\hline Corrida & FeO & CaO & SiO2 & MgO & MnO & P2O5 & Al2O3 & $\begin{array}{c}\text { Bas } \\
\text { Esc }\end{array}$ & $\begin{array}{c}\text { Peso } \\
\text { Escória(kg/taço) }\end{array}$ \\
1 & 21,09 & 48,20 & 14,70 & 8,10 & 3,99 & 2,03 & 1,89 & 3,28 & 126,0 \\
2 & 25,60 & 43,90 & 13,00 & 10,00 & 4,31 & 1,61 & 1,58 & 3,38 & 119,4 \\
3 & 22,22 & 47,50 & 14,90 & 7,50 & 4,05 & 2,05 & 1,78 & 3,19 & 114,4 \\
4 & 17,20 & 45,70 & 18,00 & 10,70 & 4,24 & 2,44 & 1,72 & 2,54 & 97,5 \\
5 & 21,66 & 44,60 & 15,40 & 10,20 & 4,41 & 2,49 & 1,24 & 2,90 & 98,0 \\
6 & 21,94 & 46,70 & 14,60 & 8,80 & 4,33 & 2,09 & 1,54 & 3,20 & 105,7 \\
7 & 28,77 & 47,30 & 9,10 & 9,40 & 2,88 & 1,18 & 1,37 & 5,18 & 160,0 \\
8 & 19,97 & 50,90 & 13,90 & 8,70 & 3,62 & 1,96 & 0,95 & 3,66 & 187,7 \\
9 & 24,44 & 46,20 & 13,20 & 8,70 & 4,05 & 2,16 & 1,25 & 3,50 & 117,1 \\
10 & 22,27 & 47,70 & 14,40 & 7,30 & 4,29 & 2,17 & 1,87 & 3,31 & 100,7 \\
11 & 15,03 & 52,80 & 16,50 & 9,50 & 3,36 & 1,74 & 1,07 & 3,20 & 151,7 \\
12 & 17,00 & 47,40 & 17,50 & 8,70 & 4,51 & 2,09 & 2,80 & 2,71 & 104,0 \\
13 & 25,54 & 45,40 & 13,00 & 8,40 & 4,26 & 1,94 & 1,46 & 3,49 & 115,6 \\
14 & 23,44 & 44,20 & 13,50 & 10,40 & 4,80 & 1,77 & 1,89 & 3,27 & 116,5 \\
15 & 18,95 & 47,60 & 17,00 & 8,40 & 3,92 & 2,40 & 1,73 & 2,80 & 98,2 \\
16 & 27,64 & 42,20 & 14,40 & 7,60 & 4,80 & 2,15 & 1,21 & 2,93 & 121,8 \\
\hline Média & 22,05 & 46,77 & 14,57 & 8,90 & 4,11 & 2,02 & 1,58 & 3,28 & 120,9 \\
\hline
\end{tabular}

\section{RESULTADOS E DISCUSSÃO}

\subsection{Relação entre (Lp), \%Peq e y $\mathrm{P}_{2} \mathrm{O}_{5}$ na Eficiência da Desfosforação}

A Tabela 4 mostra os valores do $L p, \gamma_{2} \mathrm{P}_{2} \mathrm{O}_{5}$ e $\% \mathrm{P}_{\text {Equilibrio }}$ calculado pelo Facstage usando FToxid. 
Tabela 4. Dados de equilíbrio termodinâmico das simulações usando o FactSage (com FToxid)

\begin{tabular}{ccccc}
\hline Corridas & \%Pequilíbrio & Lp equilíbrio & $(-) \log \gamma \mathbf{P 2 O 5}$ & $\mathbf{n}(\%)$ \\
$\mathbf{1}$ & 0,002737 & 323,88 & 17,81 & 85,1 \\
$\mathbf{2}$ & 0,002152 & 326,67 & 17,42 & 83,3 \\
$\mathbf{3}$ & 0,002553 & 350,60 & 17,63 & 85,9 \\
$\mathbf{4}$ & 0,003861 & 275,93 & 17,01 & 81,9 \\
$\mathbf{5}$ & 0,002651 & 410,10 & 17,27 & 80,3 \\
$\mathbf{6}$ & 0,002427 & 376,04 & 17,70 & 84,4 \\
$\mathbf{7}$ & 0,001571 & 327,95 & 18,06 & 84,3 \\
$\mathbf{8}$ & 0,00254 & 336,87 & 18,14 & 87,6 \\
$\mathbf{9}$ & 0,002403 & 392,40 & 17,67 & 85,9 \\
$\mathbf{1 0}$ & 0,002651 & 357,45 & 17,71 & 83,8 \\
$\mathbf{1 1}$ & 0,002578 & 294,66 & 18,30 & 85,9 \\
$\mathbf{1 2}$ & 0,00453 & 201,43 & 17,17 & 75,7 \\
$\mathbf{1 3}$ & 0,00254 & 333,46 & 17,45 & 84,6 \\
$\mathbf{1 4}$ & 0,002208 & 350,06 & 17,55 & 80,8 \\
$\mathbf{1 5}$ & 0,003483 & 300,88 & 17,30 & 78,1 \\
$\mathbf{1 6}$ & 0,003091 & 303,67 & 16,62 & 78,7 \\
\hline
\end{tabular}

Quando analisados os dados obtidos no equilíbrio metal-escória, os constituintes da atividade da escória têm influência significativa na \%DeP, com isso $\mathrm{a}_{\mathrm{CaO}} \mathrm{e} \mathrm{a}_{\mathrm{FeO}}$ são as mais significativas. No entanto, o efeito negativo da $a_{\mathrm{FeO}}$ na \% De-P é também observada. Ambos $\mathrm{a}_{\mathrm{FeO}}$ como $\mathrm{a}_{\mathrm{SiO} 2}$ são fatores que aumentam levando à redução\% De-P. Este efeito está relacionado com a capacidade de fixação de fósforo na escória.

A partir da Figura 1A, pode ser visto que a eficiência de desfosforação é favorecida pela diminuição da yP2O5 e aumento da $\mathrm{a}_{\mathrm{CaO}}$, devido a uma maior capacidade de fixação de fósforo na escória. O efeito destas variáveis pode também ser verificadas pelas Equações 5 e 6 . Na Equação 5, observa-se que o aumento da $\mathrm{a}_{\mathrm{caO}}$ e da a3CaO.P2O5, ocorre uma diminuição do teor de equilíbrio de fósforo no metal. Voltando agora à Equação 6, segue-se que, com a redução do teor de fósforo remanescente, há um aumento na expressão "[\% Pi] - [\% Peq]" e na \% De-P.

$$
\begin{array}{r}
\ln [\% \mathrm{P}]=\frac{1}{2} \ln a_{\left(3 \mathrm{CaO} . \mathrm{P}_{2} \mathrm{O}_{5}\right)}+\frac{\Delta G^{0}}{2 R T}-\frac{5 \mu_{\mathrm{O}_{2}}}{4 R T}-\frac{3}{2} \ln a_{(\mathrm{CaO})}-\ln f_{P} \\
-\frac{d[\% \mathrm{P}]}{d t}=k\left\{\left[\% P_{i}\right]-\left[\% P_{e q}\right]\right\}
\end{array}
$$




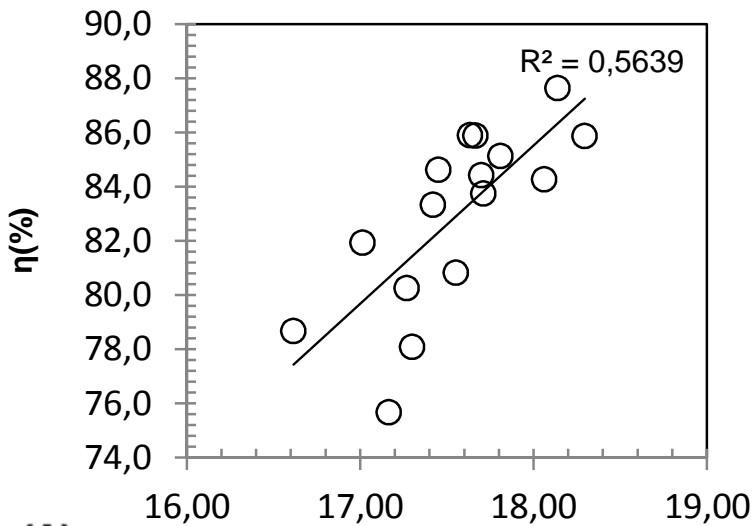

(A)

(-) $\log$ yP2O5

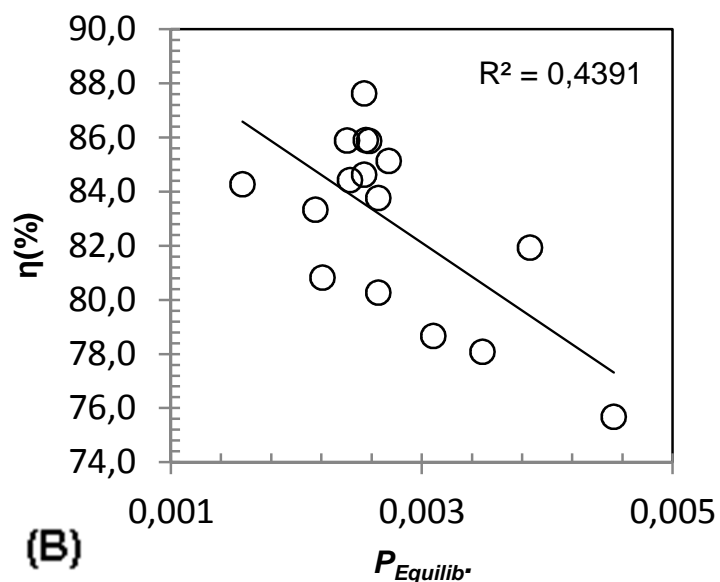

Figura 1. Eficiência da desfosforação correlacionada com o coeficiente de partição do fósforo e o fósforo de equilíbrio.

Finalmente, a eficiência de desfosforação pode ser correlacionada com um parâmetro utilizado na avaliação da eficiência da desfosforação, o coeficiente de partição de fósforo. Tomando em consideração que o coeficiente de partição é uma função da quantidade de equilíbrio de fósforo, como pode ser visto na Figura 1B, segue-se que, apesar da diferença entre os valores, o equilíbrio de correlação de fósforo com \% DeP é muito semelhante.

De acordo com este resultado, é necessário avaliar a eficiência da desfosforação, utilizando uma certa precisão para o tipo de parâmetro eficiência da desfosforação ou Lp. Isto é necessário porque o parâmetro escolhido pode realçar claramente o comportamento deste processo. Caso contrário, as mudanças na prática operacional podem estar sendo avaliadas incorretamente, comprometendo o resultado esperado após as alterações. Devido ao pequeno número de dados, essa discussão não seria possível neste trabalho, e seria necessário um estudo maior. Entre os modelos de partição na literatura [8-10], o modelo de Healey foi o melhor no que diz respeito aos resultados correlacionando com 0 Lp obtido por simulação do software termodinâmica computacional. Estes resultados são mostrados na Figura 2.

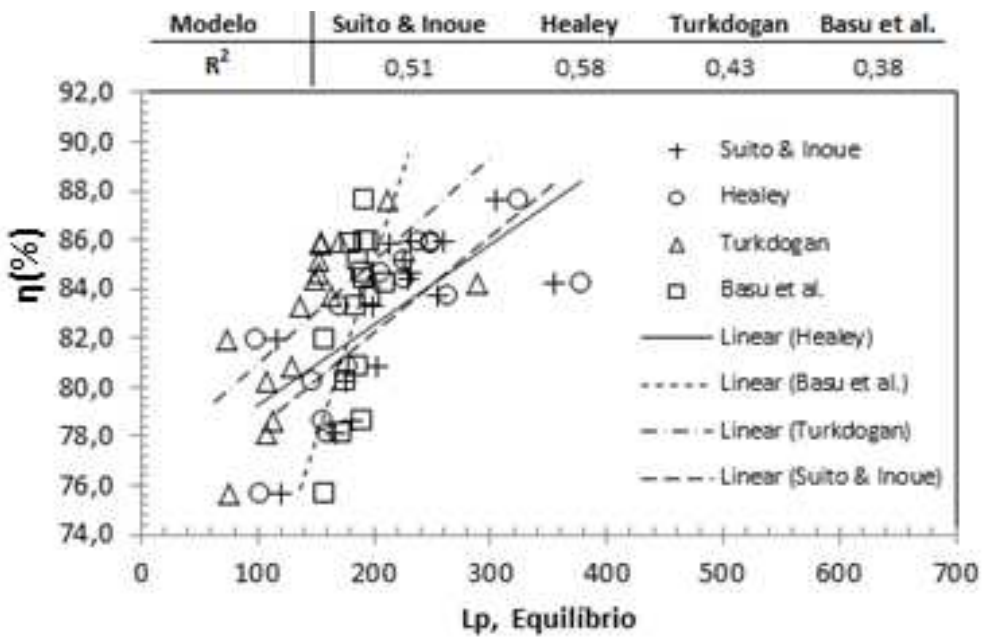

Figura 2. Resultados de Lp encontrado dos modelos termodinâmicos da literatura. 


\subsection{Relação entre $\mathrm{a}_{\mathrm{CaO}}$, $\mathrm{a}_{\mathrm{FeO}}$, Porcentagem de Escória Líquida e Eficiência da Desfosforação Determinada pelo FactSage Usando FToxid}

Utilizando o FactSage (FToxid), foi feita uma simulação para caracterizar a escória de acordo com a temperatura do sopro final. Os resultados da composição química e as fases presentes podem ser vistos na Tabela 5 .

Nenhuma das escórias houve a formação de fases sólidas $2 \mathrm{CaO} . \mathrm{SiO}_{2}, 3 \mathrm{CaO} . \mathrm{SiO}_{2} \mathrm{e}$ $2 \mathrm{CaO} . \mathrm{SiO}_{2}-3 \mathrm{CaO} . \mathrm{P}_{2} \mathrm{O}_{5}$. A atividade $\mathrm{MgO}$ foi de $1 \mathrm{em}$ todas as escórias, ou seja, todas as escórias estavam saturadas em MgO.

Tabela 5. Caracterização das escórias usando o Factsage (FToxid)

\begin{tabular}{cccccc}
\hline Corridas & $\mathbf{a}_{\mathrm{FeO}}$ & $\mathbf{a}_{\mathrm{CaO}}$ & $\begin{array}{c}\text { Escória } \\
\text { Líquida } \\
(\%)\end{array}$ & $\mathbf{n}$ (Poise) & $\mathbf{n ( \% )}$ \\
\hline $\mathbf{1}$ & 0,13 & 0,83 & 96,31 & 0,41 & 85,1 \\
\hline $\mathbf{2}$ & 0,16 & 0,68 & 94,86 & 0,42 & 83,3 \\
\hline $\mathbf{3}$ & 0,14 & 0,74 & 97,07 & 0,39 & 85,9 \\
\hline $\mathbf{4}$ & 0,17 & 0,51 & 94,17 & 0,53 & 81,9 \\
\hline $\mathbf{5}$ & 0,17 & 0,62 & 94,45 & 0,48 & 80,3 \\
\hline $\mathbf{6}$ & 0,14 & 0,76 & 95,54 & 0,44 & 84,4 \\
\hline $\mathbf{7}$ & 0,12 & 1,00 & 91,17 & 0,46 & 84,3 \\
\hline $\mathbf{8}$ & 0,11 & 1,00 & 90,63 & 0,52 & 87,6 \\
\hline $\mathbf{9}$ & 0,15 & 0,78 & 95,67 & 0,41 & 85,9 \\
\hline $\mathbf{1 0}$ & 0,14 & 0,78 & 97,14 & 0,40 & 83,8 \\
\hline $\mathbf{1 1}$ & 0,10 & 1,00 & 88,23 & 0,66 & 85,9 \\
\hline $\mathbf{1 2}$ & 0,14 & 0,59 & 96,43 & 0,79 & 75,7 \\
\hline $\mathbf{1 3}$ & 0,15 & 0,72 & 96,39 & 0,70 & 84,6 \\
\hline $\mathbf{1 4}$ & 0,15 & 0,70 & 94,17 & 0,96 & 80,8 \\
\hline $\mathbf{1 5}$ & 0,15 & 0,62 & 96,24 & 0,80 & 78,1 \\
\hline $\mathbf{1 6}$ & 0,21 & 0,45 & 98,19 & 0,71 & 78,7 \\
\hline
\end{tabular}

A Figura 3 mostra que, para os valores apresentados neste estudo, com o aumento da percentagem de escória líquida diminui a \%DeP. Ela também mostra que, com o aumento da viscosidade da escória, a \%DeP aumentou. No entanto, a correlação entre esses parâmetros e $\eta$ foi baixa.
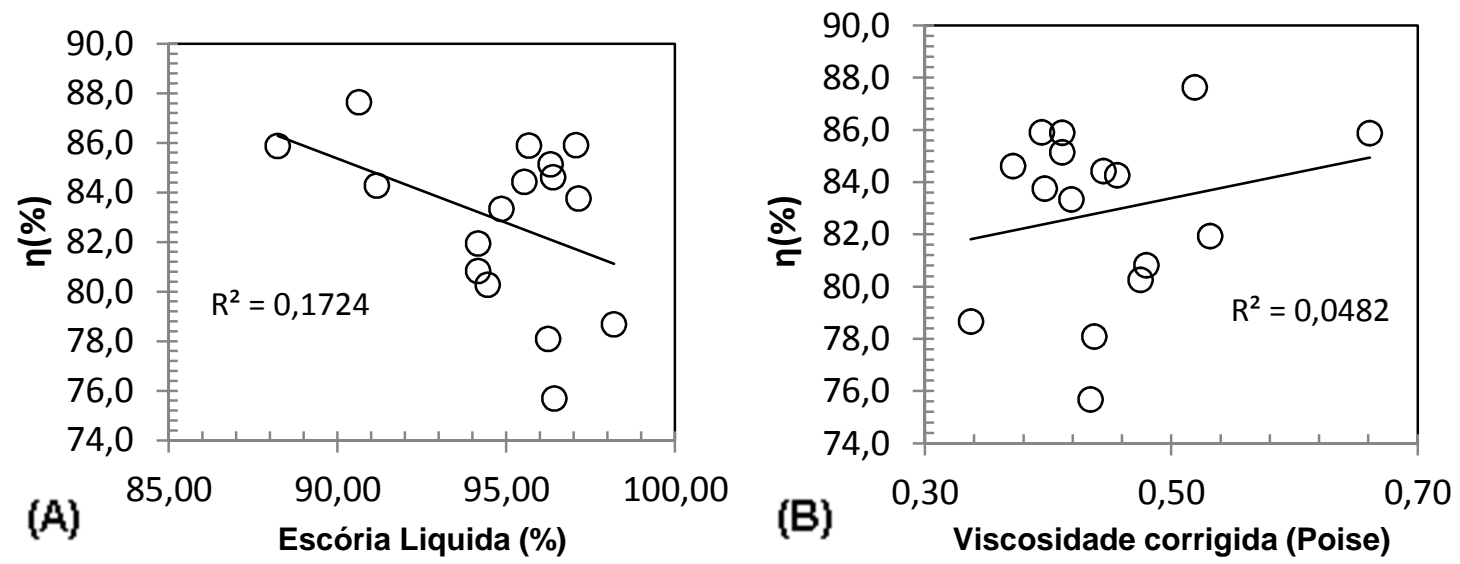

Figura 3. Eficiência da desfosforação correlacionada com a escória líquida e a viscosidade. 
Foi verificado que os resultados apresentados nos gráficos da Figura 3 não foram os esperados. Isto porque, uma vez que a reação de formação de $\mathrm{P}_{2} \mathrm{O}_{5}$ ocorre na escória líquida, uma maior percentagem de líquido e uma viscosidade mais baixa da escória deve favorecer a De-P. No entanto, um aumento da porcentagem de escória líquida e uma diminuição na viscosidade diminui a atividade de $\mathrm{CaO}$, como mostrado na Figura 4. Este, em seguida, causou uma diminuição na eficiência da desfosforação. Devido a estes resultados, é evidente que o fator que tem a maior influência sobre DeP é a atividade do $\mathrm{CaO}$. Assim, é possível analisar a influência da porcentagem de escória líquida e $\mathrm{aFeO}$ na $\mathrm{aCaO}$, como pode ser observado na Figura 4.
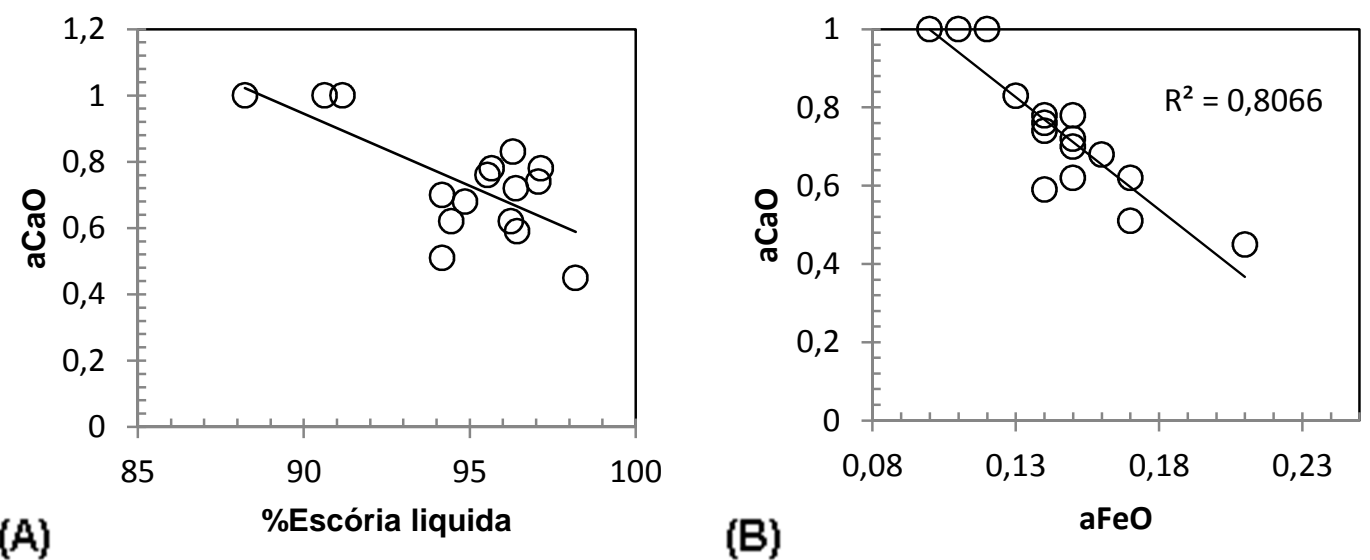

Figura 4. Influência da \% de escória líquida e aFeO na aCaO.

Observando a Figura 4 temos que uma maior porcentagem de líquido e atividade do $\mathrm{FeO}$ diminuem a atividade do $\mathrm{CaO}$. Porém de acordo com as Equações 5 e 6, quanto maior a $\mathrm{aFeO}$ e porcentagem de líquidos melhor a desfosforação. Assim, esses valores devem ser os maiores possíveis desde que não se tenha uma diminuição da atividade da $\mathrm{CaO}$.

Dessa forma, para uma analise mais eficiente da desfosforação, criou-se o fator de desfosforação $(F)$, sendo:

Fator de Desfosforação $(F)=a C a O+a F e O+\%$ Liquido $* 0,001(7)$

Correlacionando o fator de desfosforação com a eficiência da desfosforação foi possível obter um $R^{2}(0,75)$ elevado, visto que os dados analisados são dados industriais, como pode ser observado na figura 5. 


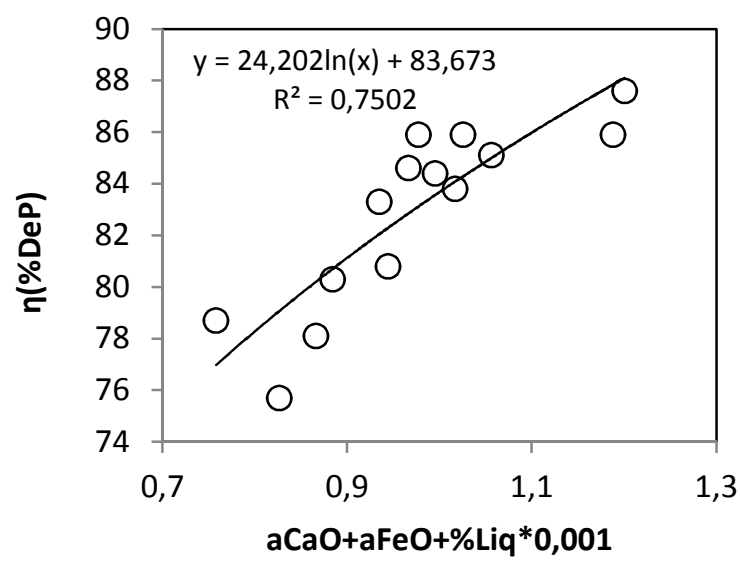

Figura 5. Correlação do fator de desfosforação com a eficiência da desfosforação.

Como este valor de $\mathrm{R}^{2}$ pode ser considerado alto, é possível então criar um modelo para prever $\eta$ usando a equação da linha de tendência gerada na figura 5:

$$
\begin{array}{r}
y=24,202 \ln (x)+83,673 \\
\eta(\% \text { DeP })=24,202 \ln (F)+83,673
\end{array}
$$

Um dos efeitos da utilização da termodinâmica computacional consiste em prever a eficácia de escórias desfosforantes. Para a análise da Equação 5 e 6, pode ser visto que as escórias mais eficazes são aquelas que tem ao mesmo tempo:

- acao é igual à 1;

- Maior quantidade de líquido possível;

Isto assegura uma atividade $3 \mathrm{CaO} \cdot \mathrm{P}_{2} \mathrm{O}_{5}$ inferior e aumento da quantidade de $\mathrm{FeO}$, desde que quantidades maiores de $\mathrm{FeO}$ aumentem a quantidade de líquido e 0 potencial de oxigênio, para além de reduzir a viscosidade da escória. No entanto, no que diz respeito ao segundo ponto, a porcentagem de FeO pode não ser tão alta, o que diminui para menos de $1 \mathrm{a} \mathrm{caO}$.

Pela análise da Tabela 5 pode-se notar que as escórias que tiveram $\mathrm{a}_{\mathrm{CaO}}=1$ (corridas 7,8 e 11), obtiveram alto rendimento de desfosforação, apesar de não ter a maior porcentagem de líquido. A eficiência desfosforação (\%DeP) da escória 7, 8 e 11 foi maior do que da escória 10 e 16, as quais mostraram maior quantidade de fase líquida na escória. Assim, pode-se inferir que as escórias 7, 8 e 11 tiveram maior quantidade de fase líquida, mantendo-se a atividade do $\mathrm{CaO}=1$, tendo uma eficiência maior.

Assim, pode concluir-se que a escória mais eficaz é a capaz de manter $\mathrm{a}_{\mathrm{caO}}=1$ ao longo do processo com uma maior quantidade de fase líquida. Um excesso de $\mathrm{CaO}$ irá gerar fases sólidas contendo $\mathrm{CaO}$. Estes sólidos, por consequência, irão prejudicar a desfosforação diminuindo a quantidade de liquido e aumentando a viscosidade da escória.

No entanto, como o FTOXID não apresentou a formação das fases sólidas citadas na introdução deste trabalho, foram feitas outras simulações usando outro banco de dados chamado GTOXID. 


\subsection{Caracterização das Escórias Usando o Factsage (GTOXID)}

Como as simulações com o FTOXID não apresentaram fases sólidas $2 \mathrm{CaO} \mathrm{SiO}_{2}$, $3 \mathrm{CaO} . \mathrm{SiO}_{2}$ e $\mathrm{Ca}_{7} \mathrm{P}_{2} \mathrm{Si}_{2} \mathrm{O}_{16}-7 \mathrm{CaOP}_{2} \mathrm{O}_{5}$, foram realizadas outras simulações utilizando o banco de dados GTOXID, desenvolvido especificamente para tratar a DeP. Com esta base de dados é possível analisar a influência das fases mencionadas.

A Tabela 6 mostra os resultados obtidos com o Factsage usando o banco de dados GTOXID.

Tabela 6. Resultados obtidos com o Factsage usando o banco de dados GTOXID

\begin{tabular}{|c|c|c|c|c|c|c|c|c|c|c|c|}
\hline $\begin{array}{l}\text { 음 } \\
\text { 금. } \\
\text { 읍 }\end{array}$ & $\frac{\circ}{\frac{\circ}{\Gamma}}$ & 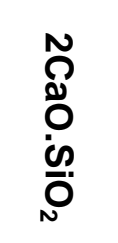 & 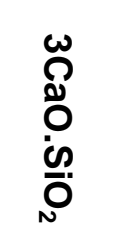 & 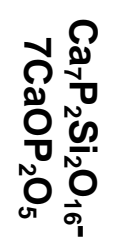 & ْి & 3a & $\begin{array}{l}\text { 울 } \\
\text { 을 } \\
\text { 웅 }\end{array}$ & $\begin{array}{l}\stackrel{\mathscr{\Omega}}{\Omega} \\
\stackrel{0}{0}\end{array}$ & 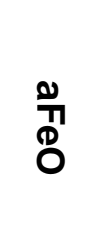 & 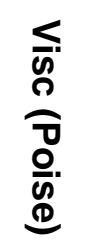 & $\frac{\overrightarrow{\widehat{0}}}{\mathrm{~d}}$ \\
\hline 1 & 62,70 & 14,29 & 12,17 & 8,23 & - & 2,22 & 37,30 & 0,86 & 0,12 & 0,23 & 85,10 \\
\hline 2 & 70,80 & 18,91 & - & 6,39 & - & 3,35 & 29,10 & 0,86 & 0,12 & 0,21 & 83,30 \\
\hline 3 & 63,70 & 20,26 & 5,64 & 8,44 & - & 1,55 & 36,29 & 0,86 & 0,12 & 0,23 & 85,90 \\
\hline 4 & 53,20 & 31,17 & - & 9,56 & - & 5,71 & 46,79 & 0,68 & 0,14 & 0,27 & 81,90 \\
\hline 5 & 57,90 & 26,40 & - & 10,57 & - & 4,78 & 42,13 & 0,76 & 0,14 & 0,24 & 80,30 \\
\hline 6 & 60,60 & 20,29 & 6,67 & 8,83 & - & 3,23 & 39,41 & 0,86 & 0,13 & 0,23 & 84,40 \\
\hline 7 & 74,80 & - & 15,48 & 4,71 & 2,26 & 2,22 & 25,18 & 1,00 & 0,12 & 0,19 & 84,30 \\
\hline 8 & 53,70 & - & 33,91 & 8,40 & - & 3,62 & 46,27 & 0,70 & 0,13 & 0,22 & 87,60 \\
\hline 9 & 65,20 & 12,49 & 10,16 & 9,13 & - & 2,62 & 34,84 & 0,86 & 0,13 & 0,22 & 85,90 \\
\hline 10 & 63,80 & 16,15 & 9,21 & 9,02 & - & 1,39 & 36,17 & 0,86 & 0,12 & 0,23 & 83,80 \\
\hline 11 & 42,90 & 5,98 & 37,88 & 7,39 & - & 5,50 & 57,01 & 0,86 & 0,12 & 0,26 & 85,90 \\
\hline 12 & 67,40 & 23,28 & - & 6,41 & - & 2,54 & 32,59 & 0,80 & 0,11 & 0,25 & 75,70 \\
\hline 13 & 71,40 & 13,69 & 4,96 & 7,83 & - & 1,61 & 28,55 & 0,86 & 0,12 & 0,20 & 84,60 \\
\hline 14 & 65,50 & 21,31 & 1,10 & 7,28 & - & 4,43 & 34,53 & 0,86 & 0,13 & 0,23 & 80,80 \\
\hline 15 & 54,90 & 30,56 & - & 10,02 & - & 3,25 & 44,19 & 0,82 & 0,13 & 0,25 & 78,10 \\
\hline 16 & 74,10 & 16,92 & - & 8,06 & - & 0,43 & 25,94 & 0,65 & 0,15 & 0,20 & 78,70 \\
\hline
\end{tabular}

É possível perceber a diferença entre os valores de fase líquida para ambos os bancos de dados do programa. Além disso, usando o GTOXID é possível observar a formação de fases sólidas, tais como $2 \mathrm{CaO} . \mathrm{SiO}_{2}, 3 \mathrm{CaO} . \mathrm{SiO}_{2}$ e $\mathrm{Ca}_{7} \mathrm{P}_{2} \mathrm{Si}_{2} \mathrm{O}_{16^{-}}$ $7 \mathrm{CaOP}_{2} \mathrm{O}_{5}$ além das fases presentes do $\mathrm{CaO}$ e $\mathrm{MgO}$ no FToxid. Mas, a quantidade de escória líquida foi pequena.

Os dados obtidos foram tratados da mesma forma que usando a base de dados FTOXID, a fim de se relacionar as propriedades da escória com \%DeP.

A Figura 6A, Figura 6B e Figura 6C mostra a influência das fases sólidas $\mathrm{CaO}$, $2 \mathrm{CaO} . \mathrm{SiO}_{2}$ e $3 \mathrm{CaO} . \mathrm{SiO}_{2}$ na eficiência da desfosforação. 


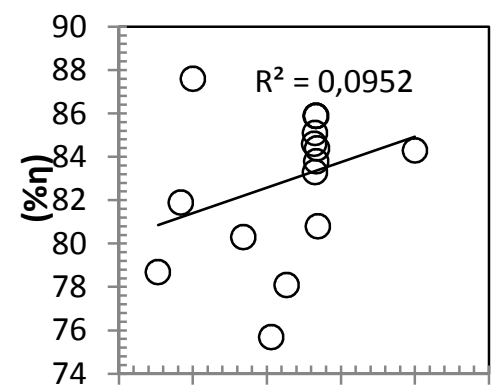

(A) $0,6 \quad 0,7 \quad 0,8 \quad 0000001$
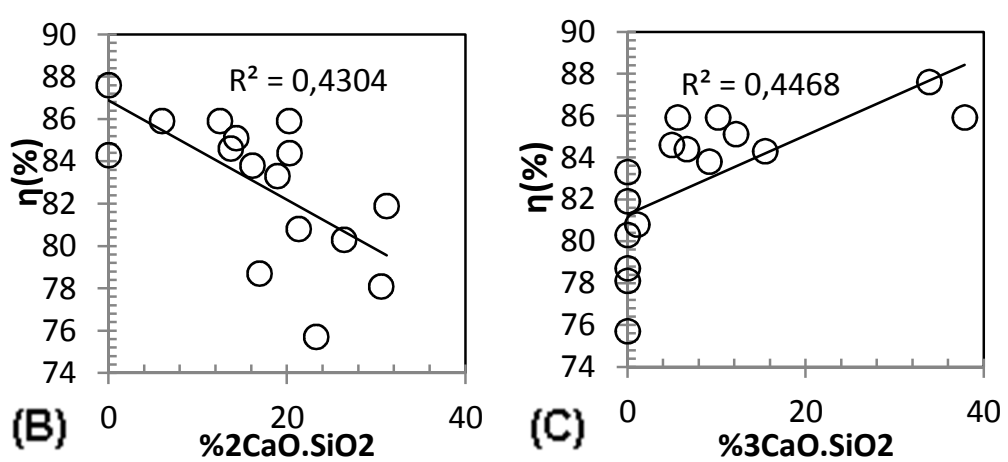

Figura 6. Influência das fases sólidas $\mathrm{CaO}, 2 \mathrm{CaO} \mathrm{SiO}_{2}$ e $3 \mathrm{CaO} . \mathrm{SiO}_{2}$ na eficiência da desfosforação.

Pela análise da Figura 6A e Figura 6C nota-se que o aumento nas atividades de $\mathrm{CaO}$ e \% $3 \mathrm{CaO} . \mathrm{SiO}_{2}$ aumenta a eficiência da desfosforação. No entanto, os valores de correlação $\left(R^{2}\right)$ são baixos. A Figura $7 A$ mostra que não é possível obter uma correlação tão boa quanto à alcançada utilizando o FTOXID para o fator de desfosforação já analisado. Assim, outra relação foi criada para as simulações com o GTOXID, sendo a eficiência da desfosforação analisada em função de todos os parâmetros que já citados que afetam a desfosforação, incluindo nesse caso, a formação das fases sólidas que o GTOXID apresenta. Dessa forma, o fator de desfosforação para as simulações com o GTOXID é dado por:

$$
F=\% \text { liquido }+a \mathrm{CaO}+a \mathrm{FeO}+\% 3 \mathrm{CaO} \cdot \mathrm{SiO}_{2}+\% \mathrm{Ca}_{7} \mathrm{P}_{2} \mathrm{Si}_{2} \mathrm{O}_{16} \cdot 7 \mathrm{CaOP}_{2} \mathrm{O}_{5}-\% 2 \mathrm{CaO} \cdot \mathrm{SiO}
$$

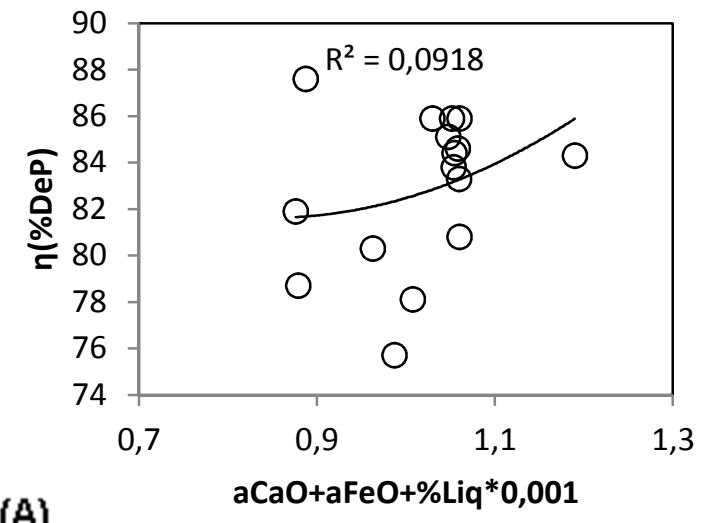

(A)

Figura 7. Correlação da eficiência com o fator de desfosforação $F$, utilizado para o FTOXID (A) e o GTOXID (B).

Nota-se na Figura 7B, que o $R^{2}(0,73)$ apresenta um valor elevado também, portanto é possível gerar uma expressão para se determinar a eficiência da desfosforação a partir da equação da linha de tendência da mesma forma que foi feita para as simulações com o FTOXID.

$$
\begin{array}{r}
y=7,2245 \ln (x)+54,22 \\
\eta(\% \text { DeP })=7,2245 \ln (F)+54,22
\end{array}
$$

Isto mostra que, ao utilizar o FactSage pode encontrar uma ligação para predizer com mais precisão a eficiência na escória da desfosforação, no entanto, a quantidade de escória líquida determinado pelo software não é exata. 


\section{CONCLUSÃO}

Com base nos dados apresentados neste trabalho pode-se concluir que:

- O teor de fósforo remanescente em aço (Peq) encontrado no FactSage está dentro do intervalo de dados de trabalho publicados na literatura;

- A eficiência de desfosforação é favorecida pelo aumento da $\mathrm{a}_{\mathrm{CaO}}, \mathrm{a}_{\mathrm{FeO}}$, e porcentagem de escória líquida, desde que não diminua a atividade do $\mathrm{CaO}$;

-Para o FTOXID o fator de desfosforação é $\mathrm{F}=\mathrm{aCaO}+\mathrm{aFeO}+\%$ liquido*0,001, obtendo-se uma correlação com a eficiência da desfosforação de 0,75 ;

- Para o GTOXID, o fator de desfosforação é:

$\mathrm{F}=\%$ liquido+aCaO+aFeO+ $\% 3 \mathrm{CaO} . \mathrm{SiO}_{2}-\% 2 \mathrm{CaO} . \mathrm{SiO}_{2}-\%\left(\mathrm{Ca}_{7} \mathrm{P}_{2} \mathrm{Si}_{2} \mathrm{O}_{16}{ }^{-}\right.$ $7 \mathrm{CaOP}_{2} \mathrm{O}_{5}$ ), obtendo-se uma correlação com a eficiência de desfosforação de 0,73 ;

- Através da utilização do FactSage pode-se encontrar uma relação mais precisa para prever a eficiência de desfosforação de diferentes escórias. O modelo mais preciso foi 0 encontrado usando 0 banco de dados FT OXID, que é: $\eta(\%)=24,202 \ln (F)+83,673$;

- A escória que apresentar no fim de sopro atividade do $\mathrm{CaO}$ igual a 1 e maior quantidade de líquido e maior atividade do FeO será a mais eficiente.

\section{Agradecimentos}

Gostaríamos de agradecer a CAPES e FAPES pela bolsa de estudos para 0 mestrado.

\section{REFERÊNCIAS}

1 Turkdogan ET. Fundamentals of Steelmaking. 1. ed. London: The Institute of Materials, 1996.

2 Turkdogan ET. Slag composition variations causing variations in steel dephosphorisation and desulphurisation in oxygen steelmaking. ISIJ International; ISSN:0915-1559, v..40. No. 9. p.827-832, 2000.

3 Suito $\mathrm{H}$, Inoue R, Takada M. Phosphorus distribution between liquid iron and $\mathrm{MgO}$ saturated slags of the system $\mathrm{CaO} \_\mathrm{MgO} \_\mathrm{FeO} \_\mathrm{SiO}_{2}$. Trans. Iron Steel Inst. Jpn., 21,p. 250-259, 1981b.

4 Swinnerton M. The influence of slag evolution on BOF dephosphorisation. University of Wollongong. Master of Engineering Thesis. Australia, 2005.

5 Lee YS, Seok SH, Jung SM, Min DJ. Viscosity of highly basic slags. ISIJ International, v. 47, No 08, p. 1090-1096, 2007.

6 Miyamoto KI, Mukawa S, Ogawa Y,Sasaki N. Improvement in hot-metal dephosphorization. NIPPON STEEL TECHNICAL REPORT No. 104, AUGUST, p. 2632, 2013.

7 Bardenheuer F, Oberhauser P. Stahl u. Eisen, 89, p.988, 1969.

8 Suito $\mathrm{H}$, Inoue $\mathrm{R}$. Thermodynamic assessment of hot metal and steel dephosphorisation with MnO-containing BOF slags. ISIJ International, n. 3, v. 35, p. 258-265, 1995a.

9 Healy G. A new look at phosphorus distribution. Journal of the Iron and Steel Institute, n. 6 , v. 208 , p. $664-668,1970$.

10 Basu S, Lahiri AK. Seetharaman S. A model for activity coefficient of P2O5 in bof slag and phosphorus distribution between liquid steel and slag. ISIJ International, n. 8, v. 47, p. 1236-1238, 2007d. 